\title{
Formation Flying Control Implementation in Highly Elliptical Orbits
}

\author{
Pedro A. Capó-Lugo* \\ NASA George C. Marshall Space Flight Center \\ Huntsville AL 35812 \\ Peter M. Bainum \\ Department of Mechanical Engineering \\ Howard University \\ Washington, D.C. 20059
}

\section{Introduction}

The Tschauner-Hempel equations are widely used to correct the separation distance drifts between a pair of satellites within a constellation in highly elliptical orbits [1]. This set of equations was discretized in the true anomaly angle [1] to be used in a digital steady-state hierarchical controller [2]. This controller [2] performed the drift correction between a pair of satellites within the constellation. The objective of a discretized system is to develop a simple algorithm to be implemented in the computer onboard the satellite. The main advantage of the discrete systems is that the computational time can be reduced by selecting a suitable sampling interval. For this digital system, the amount of data will depend on the sampling interval in the true anomaly angle [3]. The purpose of this paper is to implement the discrete Tschauner-Hempel equations and the steady-state hierarchical controller in the computer onboard the satellite. This set of equations is expressed in the true anomaly angle in which a relation will be formulated between the time and the true anomaly angle domains.

\section{Discrete Tschauner-Hempel Equations and Control System}

In reference [2], the discrete $\mathrm{TH}$ equations for a perturbed motion that defines the motion of a pair of satellites in an elliptical orbit about the Earth can be described as,

where,

$$
y(k+1)=A(k) y(k)+B(k) u(k)+\psi[y(k)]+\Gamma(f(k))
$$

$$
y(k)=\left[\begin{array}{llllll}
y_{1}(k) & y_{2}(k) & y_{3}(k) & y_{1}^{\prime}(k) & y_{2}^{\prime}(k) & y_{3}^{\prime}(k)
\end{array}\right] ; \quad()^{\prime}=d() / d f ; \quad y_{j}(k)=(1+e \cos f) x_{j}(k)
$$

$x_{1}$ is positive against the motion of the spacecraft, $x_{2}$ is positive along the radial direction, and $x_{3}$ is positive when the right handed system is completed. The $y_{j}$ equations are used as a mathematical transformation. $\psi(k)$ defines the perturbation due to the oblateness of the Earth as explained in Ref. [1]. $\Gamma_{j}(f(k))$ is the solar pressure force defined by the Karmyov theory [1]. The maneuvering spacecraft is assumed to have an applied thrust vector along the reference coordinate system, and the reference spacecraft is initially assumed to be acted on by a Newtonian gravitational force directed toward the center of the Earth. In this work, the satellites are assumed to be opaque right circular cylinders where the incident solar rays can affect the motion of the satellites.

The steady-state hierarchical control scheme was implemented with the cost function defined in Ref. [2]. In steady-state [2], the control problem can be reduced and defined in the following form,

$$
\left.y(k+1)=\left[\hat{A}(k)-\hat{B}(k) K(k)-\hat{B}(k) K_{\psi}(k)\right\rfloor y(k)+\left\lfloor\Delta f I-\hat{B} K_{p}(k)\right\rfloor \psi[y(k)]+\Gamma(f(k))\right]
$$

where $\hat{A}(k)=I+\Delta f A(k)$, and $\hat{B}(k)=\Delta f B(k)$ where I is a 6 X 6 identity matrix. $A$ is the state matrix with dimensions $6 \times 6, B(k)$ is the control matrix with dimensions $6 \times 3$, and $\psi[y(k)]$ is a $6 \times 1$ matrix. $K(k)$ is the control gain, $K_{\psi}(k)$ is the gain due to the nonlinear terms in the $\psi(y(k))$ column vector, and $K_{P}(k)$ is the gain due to the sum of the perturbations $(\psi(y(k))+\Gamma(f(k)))$.

\footnotetext{
*Ph.D., Engineer, EV41, Guidance, Navigation \& Control Systems Design \& Analysis Branch, NASA Marshall Space Flight Center, Huntsville, AL 35812; tel: 1-256-961-0275; Email: Pedro.A.Capo-Lugo@nasa.gov; Member AIAA, Member AAS.

$\dagger$ Distinguished Professor of Aerospace Engineering, Emeritus (Corresponding author: pbainum@,fac.howard.edu; tel.: 1-202-806-6612; fax: 1-202-806-5258); Fellow AIAA, Fellow AAS.
} 


\section{Implementation of the Control Problem}

In reference [2], a block diagram for the steady-state hierarchical control scheme was developed. This block diagram can be easily modeled in the computer onboard the satellite. The only problem is to relate the time, in which the thrusters will be fired, to the true anomaly angle. This relationship can be obtained through the angular momentum equation used in reference [4]. This equation shows that the true anomaly angle is an increasing function of time that can be used to determine the time. By developing an interface between the time, the sample separation distance and velocity, and the block diagram of the steady-state controller, the computer onboard the satellite can perform all the necessary computations to correct the separation distance conditions in a short period of time.

\section{Results}

Figure 1 shows the control solution for the drift correction [2] for a set of initial conditions when the eccentricity is equal to 0.8 . In Figure 1, the separation distance and velocity drifts are corrected before the pair of satellites reaches the perigee point. In addition, the control effort shows a minimum consumption of thrust. In this problem, the time scale has been obtained from the angular momentum equation. This time scale shows when the thrusters should be fired to correct the separation distance drifts.

\section{Conclusion}

The present work contributes for the first time the implementation of the Tschauner-Hempel equations an $\mathrm{d} t \mathrm{~h}$ e $\mathrm{s} \mathrm{t}$ e a d y-state hierarchical control scheme in

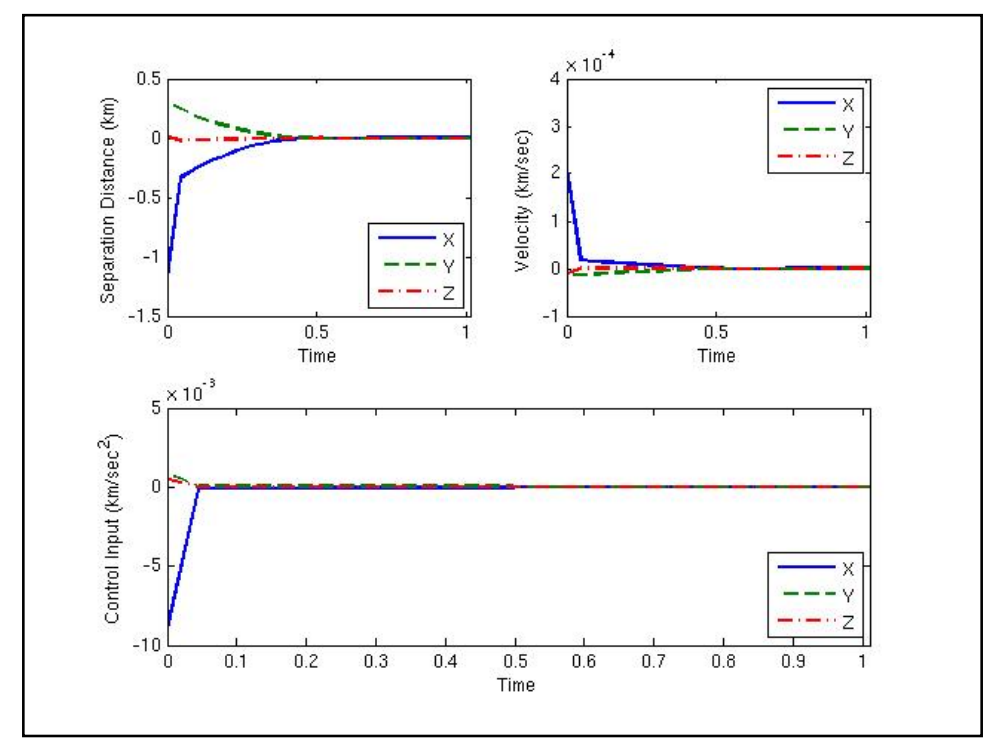

Figure 1. Drift Correction

the time domain. This discretized system can be easily implemented in the computer onboard the satellite with a simple control algorithm. The computational process will be performed in a short period of time because of the relationship determined between the time and the true anomaly angle. In this way, the controller can perform the desired maneuvers for the correction of the separation distance and velocity drifts for a pair of satellites.

\section{References:}

1. Capó-Lugo, Pedro A., and Bainum, Peter M., Solar Pressure Effects for a Constellation in Highly Elliptical Orbit, $18^{\text {th }}$ AAS/AIAA Space Flight Mechanics Conference, Galveston, Texas, January 2731, 2008, Paper No. AAS 08-190.

2. Capó-Lugo, Pedro A., and Bainum, Peter M., Steady-State Hierarchical Control for the Drift Correction of a Constellation in Highly Elliptical Orbits, F. Landis Markley Astronautics Symposium, Cambridge, Maryland, June 29 - July 2, 2008, Paper No. AAS 08-268.

3. Capó-Lugo, Pedro A., and Bainum, Peter M., Digital LQR Active Control Schemes to Satisfy the Separation Distance Constraints of the NASA Benchmark Tetrahedron Constellations, AIAA/AAS Astrodynamics Specialist Conference and Exhibit, Keystone, Colorado, August 21-24, 2006, Paper No. AIAA-2006-6014.

4. Carter, T., and Humi, M., Fuel-Optimal Rendezvous Near a Point in General Keplerian Orbit, Journal of Guidance, Control, and Dynamics, Vol. 10, No. 6, Nov—Dec 1987, Pages 567-573. 